\title{
La objeción de conciencia en México: alcances y limitaciones en materia de bioética
}

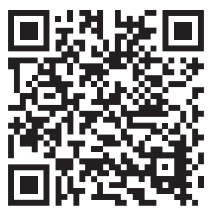

\section{Conscientious objection in Mexico: scope and limitations in bioethics}

\author{
Martha Patricia Hernández Valdez*
}

\section{RESUMEN}

La objeción de conciencia subraya un antagonismo frente a un aspecto de contenido regulativo que puede ser de carácter jurídico, cultural, social, laboral, económico, psicológico o espiritual, que hace imposible la construcción de un ordenamiento de bienestar de la persona individual. Al respecto, uno de los fundamentos antropológicos de la objeción de conciencia se establece a través del deber de dirigir la conducta hacia el bien, evitando el mal. En la dimensión legal, se acoge en el derecho fundamental de libertad ideológica, de conciencia y religiosa, como parte del reconocimiento universal de la dignidad humana que se encuentra previsto en disposiciones constitucionales mexicanas. La objeción de conciencia en sí misma no representa la solución a los extraordinarios dilemas bioéticos que enfrenta el país en la actualidad. Por ello, es fundamental la creación de estructuras jurídicas que den sustento a sus promulgaciones generales, de tal forma que no sólo exista el reconocimiento de estos derechos, sino que también se facilite el acceso a los mismos.

Palabras clave: Objeción de conciencia, alcances, limitaciones, bioética.

\begin{abstract}
The conscientious objection underlines an antagonism against an aspect of regulatory content that can be legal, cultural, social, labor, economic, psychological or spiritual, making impossible the individual well-being. In this regard, one of the anthropological foundations of this phenomenon is the human behavior towards good, avoiding evil. In the legal dimension, it embraces the fundamental right of ideological freedom, as part of the universal recognition of human dignity. Conscientious objection itself does not represent the solution to the extraordinary bioethical dilemmas. Therefore, it is essential to create legal structures that support general promulgations, in order to guaranteed recognition and the access of existed rights.
\end{abstract}

Keywords: Conscientious objection, achievements, limitations, bioethics.

* Profesora Investigadora de la Cátedra de Infertilidad «Margarita Lamas de Abad» de la Facultad de Bioética, Universidad Anáhuac México Norte. Estado de México.

Recibido: 11/11/2019. Aceptado: 14/02/2020.

Citar como: Hernández VMP. La objeción de conciencia en México: alcances y limitaciones en materia de bioética. Arch Inv Mat Inf. 2020;11(1):32-37. https://dx.doi.org/10.35366/100327 


\section{INTRODUCCIÓN}

La salud ha sido señalada por organismos internacionales como un elemento fundamental del progreso social y reconocimiento de la dignidad humana, teniendo en cuenta que todos y cada uno de los individuos activos son sujetos de su protección, tanto si se trata del personal de salud como de los pacientes y sus familias. En este sentido, los razonamientos actuales en materia de bioética poseen una importante relevancia para la clarificación de los dilemas médicos, y es precisamente la objeción de conciencia un ejercicio de la autonomía en el contexto de la atención médica que toma en cuenta la situación social, económica y de salud en el mundo, considerando sus implicaciones éticas. ${ }^{1,2}$

En la actualidad, ${ }^{3}$ la práctica de la objeción de conciencia en el mundo es creciente: $70 \%$ de quienes ejercen la ginecología en Italia y $10 \%$ de una muestra aleatoria en el Reino Unido han declarado objeción de conciencia. En Brasil, en los casos de aborto permitidos por la ley a las víctimas de violación, la objeción de conciencia es algo constante entre los profesionistas de la salud, donde llega a ser de más de $40 \%$ de objetores por la incertidumbre que gran cantidad de ellos siente al desconocer si la solicitante de los servicios efectivamente fue víctima de ese delito, siendo así que más del $80 \%$ solicitan pruebas relativas a la violación, que no son un requisito sine qua non exigido por la ley. Es importante mencionar que la objeción de conciencia no es un fenómeno exclusivo de médicos y enfermeras: $25 \%$ de farmaceutas en ciudades de Estados Unidos se han amparado en este derecho para no vender anticonceptivos de diversos tipos. ${ }^{3}$

El presente estudio tiene como objetivo analizar las consecuencias del reconocimiento de la objeción de conciencia como un derecho humano de los trabajadores de la salud, tanto del sector público como (privado) colectivo, con la finalidad de comprender sus alcances y limitaciones.

\section{LA OBJECIÓN DE CONCIENCIA EN MÉXICO: ALCANCES Y LIMITACIONES EN MATERIA DE BIOÉTICA}

La objeción de conciencia es definida conceptualmente como el rechazo a someterse a una norma o a una disposición de ley que se considera injusta, en cuanto que se opone a la ley natural, contraria al respeto de la vida y dignidad humana. De este modo, se refiere a la imposición de las convicciones personales que impiden cumplir un decreto jurídico social impuesto, construyéndose así como un recurso de oposición que tiene sentido cuando se ha de admitir que el decreto legal no es justo porque atenta contra un derecho fundamental claramente observable como lo es la vida y la integridad física. ${ }^{4}$
La objeción de conciencia subraya un antagonismo frente a un aspecto de contenido regulativo que puede ser de carácter jurídico, cultural, social, laboral, económico, psicológico o espiritual, que hace imposible la construcción de un ordenamiento de bienestar de la persona individual. Al reiterar que la conciencia se manifiesta en la persona singular, la libertad de su manifestación no tiene por titular a comunidades o grupos, y tampoco debe lesionar ningún bien protegido como el orden público y los derechos fundamentales de otros ciudadanos. Es por ello que la correcta regulación que guíe las actuaciones de los profesionistas involucrados es un tema crítico.

Para comprender los matices de este acto humano, se señalan las características de la objeción de conciencia debidamente fundamentados.'

1. Su ejercicio implica un comportamiento omisivo. Es decir, pacífico, sin objetivos políticos y de carácter individual sin extensión social que provoque la necesidad de un cambio legislativo. ${ }^{5}$

2. La objeción de conciencia se fundamenta en razones religiosas, éticas, morales, espirituales o axiológicas (que son precisamente configuraciones de la conciencia). La conciencia sitúa a la persona frente a un deber de coherencia con sus convicciones producto de la intelectualización y racionalización de eventos que dan forma a creencias de cualquier tipo ya mencionado; de esta manera, el valor que prevalece es la afirmación de la unidad inmanente de la persona. ${ }^{6}$

3. La objeción de conciencia deriva del principio fundamental de la libertad de conciencia, debidamente expresada en la Constitución Política de los Estado Unidos Mexicanos y en otros ordenamientos jurídicos, siendo importante comprender que la objeción de conciencia es un derecho anterior al Estado e intrínseco a la dignidad humana.

4. La objeción de conciencia puede ser reconocida condicional o incondicionalmente. Como se analizará más adelante, el objetor posee la obligación ética de ser examinado, sin ser objeto de discriminación o persecución, en la medida en que ello sea posible.

5. El reconocimiento expreso de la objeción de conciencia genera por su naturaleza el completo rechazo a una sanción o discriminación por el hecho de ser objetor. Una vez reconocida la objeción de

\footnotetext{
Cuando se pretende modificar las normas establecidas, se instaura la figura de desobediencia civil, que ciertamente se fundamenta muchas veces en similares motivos de conciencia. Sin embargo, es recomendable reconocer con los alcances y limitaciones de la objeción de conciencia, la diferenciación con esta forma de desacuerdo político.
} 
consciencia de un trabajador, cualquier evidencia de rechazo hacia su persona supondría un menoscabo al reconocimiento de su dignidad. ${ }^{7}$

El deber dirigir la conducta hacia el bien, evitando el mal, constituye uno de los fundamentos antropológicos de la objeción de conciencia. En cuanto a su dimensión legal, se acoge en el derecho fundamental de libertad ideológica, de conciencia y religiosa, como parte del reconocimiento universal de la dignidad humana que se encuentra previsto en disposiciones constitucionales mexicanas.

Como se ha aclarado, en sentido lógico y ontológico, los derechos fundamentales de libertad ideológica, de conciencia y religiosa son ciertamente anteriores al Estado, quien los ha reconocido pero no otorgado, por lo que no significan un privilegio de los ciudadanos porque su naturaleza no está en la sociedad, sino en el individuo mismo. Por tanto, ningún derecho humano está sujeto a que el Estado lo otorgue ni derogue, pero sí a que lo reconozca, proteja y regule; en concordancia, el ámbito internacional ha reconocido este derecho, verbigracia, el Pacto Internacional de Derechos Civiles y Políticos de la $\mathrm{Na-}$ ciones Unidas (ICCPR, por sus siglas en inglés) -que entró en vigencia en 1976, implementando la Declaración Universal de los Derechos Humanos de 1948señala en su Artículo 18 que:

Toda persona tiene derecho a la libertad de pensamiento, de conciencia y de religión. Nadie será objeto de medidas coercitivas que puedan menoscabar su libertad de tener o de adoptar la religión o las creencias de su elección. Los Estados Partes en el presente Pacto se comprometen a respetar la libertad de los padres y, en su caso, de los tutores legales, para garantizar que los hijos reciban la educación religiosa y moral que esté de acuerdo con sus propias convicciones.

En México existen algunas disposiciones legales que reconocen la objeción de conciencia como un derecho, a saber:

Artículo 24. CONSTITUCIÓN POLÍTICA DE LOS ESTADOS UNIDOS MEXICANOS. Toda persona tiene derecho a la libertad de convicciones éticas, de conciencia y de religión, y a tener o adoptar, en su caso, la de su agrado.

Artículo 18. LEY DE SALUD DEL ESTADO DE JALISCO. Los profesionales, técnicos, auxiliares y prestadores de servicio social que forman parte del Sistema Estatal de Salud, podrán hacer valer la objeción de conciencia y excusarse de participar en todos aquellos programas, actividades, prácticas, tratamientos, métodos o investigaciones que contravengan su libertad de conciencia con base en sus valores, principios éticos o creencias religiosas.
Artículo 59. LEY DE SALUD DEL DISTRITO FEDERAL. El médico a quien corresponda practicar la interrupción legal del embarazo y cuyas creencias religiosas o convicciones personales sean contrarias a tal procedimiento, podrá ser objetor de conciencia y por tal razón excusarse de intervenir; cuando sea urgente la interrupción legal del embarazo para salvaguardar la salud o la vida de la mujer, no podrá invocarse la objeción de conciencia. Es obligación de las instituciones públicas de salud del Gobierno garantizar la permanente disponibilidad de personal de salud no objetor de conciencia en la materia.

Norma Oficial Mexicana 046. En caso de embarazo por violación, y previa autorización de la autoridad competente, en los términos de la legislación aplicable, las instituciones públicas prestadoras de servicios de atención médica, deberán prestar servicios de aborto médico a solicitud de la víctima interesada, en caso de ser menor de edad, a solicitud de su padre y/o su madre, o a falta de éstos, de su tutor o conforme a las disposiciones jurídicas aplicables.

El 11 de mayo de 2018, el Congreso General de Ios Estados Unidos Mexicanos, adicionó el artículo 10 Bis a la Ley General de Salud (LGS) en el que se establece que el personal médico y de enfermería que forme parte del Sistema Nacional de Salud podrá ejercer la objeción de conciencia siempre y cuando la vida del paciente no se ponga o se encuentre en riesgo. Como respuesta inmediata, la Comisión Nacional de los Derechos Humanos presentó ante la Suprema Corte de Justicia de la Nación una acción de inconstitucionalidad en contra de esta adición del artículo $10 \mathrm{Bis}$ a la LGS, señalando que este pronunciamiento hace posible la violación de derechos fundamentales como la salud, integridad personal, seguridad jurídica, a la vida, libertades sexuales y reproductivas, derecho a decidir de manera libre, responsable e informada sobre el número y el espaciamiento de los hijos y el derecho al libre desarrollo de la personalidad. Por otra parte, la Comisión Nacional señala puntualmente que no se opone en forma alguna a una regulación del ejercicio de la objeción de conciencia, sino que exige de dicha regulación respetar el marco constitucional y convencional existente, de manera que garantice plenamente el respeto y la protección de los derechos fundamentales.

Este temor observado es desde luego fundado, pero no se encuentra enfocado en una petición concreta: es indispensable el desarrollo de guías de actuación y capacitación permanente para los servicios de salud para comprender las características, alcances y limitaciones de este fenómeno cuyo reconocimiento es relativamente nuevo en México, con la finalidad de no desconocer un derecho ontológicamente fundamentado que brinde un trato di- 
Tabla 1: Comparación de alcances y limitaciones generales.

Alcances generales de la objeción de conciencia

Promover la salud de todos los involucrados (pacientes, familiares, personal de salud)

Respetar la autonomía de los pacientes

Respetar la autonomía de los pacientes, su derecho a la información, a la salud y a la vida, validando sus preferencias

Proteger a quienes están dispuestos a cumplir la ley y a prestar servicios a conciencia

Encontrar un balance entre la integridad profesional y las necesidades e intereses de los pacientes

Limitaciones generales de la objeción de conciencia

Impedir prácticas indiscriminadas que frenen la prestación de servicios de salud, verbigracia, la objeción institucional Evitar las barreras para el acceso y la discriminación dirigida a los objetores o a los pacientes

Evitar daños físicos y emocionales del personal de la salud y los pacientes

Fuente: elaboración propia. ferenciado a los prestadores de servicios de salud, segregándolos.

Por otra parte, el conjunto de libertades encaminadas a proteger el derecho a creer y expresarse libremente de acuerdo con su conciencia para actuar conforme a ello ciertamente protege una esfera íntima y personal que atañe al individuo mismo; sin embargo, se insiste en que debe estar regulada en sus manifestaciones para proteger los derechos de otros. La objeción de conciencia merece por tanto las siguientes consideraciones:

1. Todos los profesionales de la salud tienen pleno derecho a poseer y manifestar sus creencias personales, derecho que también asiste a todos los pacientes o a sus tutores. ${ }^{i i i}$

2. Si en el ejercicio de estos derechos se entra en conflicto, el profesional no debe imponer sus propias convicciones ni desconocer las decisiones del paciente, enfocando siempre su actuar profesional hacia el reconocimiento de la dignidad humana a través de la validación. iv

3. Las creencias personales de quienes participan en los servicios de salud no pueden convertirse

\footnotetext{
ii La objeción de conciencia de los tutores de menores de edad o personas en estado de interdicción merece un manejo especial, a razón de no excluir al tutorado en la toma de decisiones a través de la escucha activa de sus deseos (si es posible) y de la consideración de su participación en cumplimiento del reconocimiento de su dignidad.

iii La validación se refiere a una estrategia de aceptación incondicional de los pensamientos, sentimientos y emociones del individuo que se ejerce con la escucha activa e intercambio de posibilidades en su tratamiento.

iv La validación se refiere a una estrategia de aceptación incondicional de los pensamientos, sentimientos y emociones del individuo que se ejerce con la escucha activa e intercambio de posibilidades en su tratamiento.
}

en obstáculo para el acceso a la protección de su bienestar.

En México, la problemática actual sobre la objeción de conciencia conlleva más que la protección o el reconocimiento de un derecho fundamental al hábito de reflexionar acerca de las propias creencias con el reconocimiento intrínseco de sus limitaciones, además del respeto a la pluralidad de afirmaciones de naturaleza diversa con el reconocimiento de las diferencias entre preceptos éticos y morales, conforma la distinción entre intimidación y expresión asertiva, descalificación, validación y negación del acceso a los servicios de salud y la protección integral de la salud.

Este pensamiento desde luego requiere importantes transformaciones en el ejercicio de las profesionales ligadas a la protección de la salud. El cambio de una atención vertical (paternalista y autoritaria) hacia un modelo horizontal y dialógico basado en la prevención, paliación, atención integral y ayuda al bienestar integral devela una faceta que obliga al Estado a reconocer al personal de salud como seres humanos merecedores de todos los derechos y obligaciones.

Al ser la objeción de conciencia un riesgo individual, que debe manifestarse cuando el profesionista es vinculado a alguna institución sin ser un requisito que discrimine o limite su contratación, se evidencia la participación de toda la estructura que regula y contrata a los trabajadores, comprometiéndola a conocer de fondo todas las características de la objeción de conciencia en México para proteger los derechos que le subyacen, siendo de vital importancia establecer sus alcances y limitaciones, propuestas en la Tabla 1.

Las generalidades antes mencionadas ponen de manifiesto los elementos necesarios para la protección del derecho a la objeción de conciencia: dis- 
ponibilidad (desarrollo de un sistema de salud que preserve este concepto en todos sus usuarios y prestadores de servicios profesionales con acceso a procedimientos de forma rápida y expedita), accesibilidad (promoción de información oportuna y suficiente para asegurar la protección a la conciencia en conjunto con el acceso a los servicios de salud) y calidad (se refiere a la oportunidad del servicio en cumplimiento de los requisitos legales, el respeto a la autodeterminación y la creación de un entorno seguro para los pacientes y los profesionales de la salud).

Con los elementos anteriormente señalados se recomienda, a través de las instancias legales correspondientes y (si ello no fuera posible) a través de programas de capacitación específica con las contribuciones de los expertos en la materia, que se ahonde en las características que dan forma a la objeción de conciencia. ${ }^{8}$

1. La objeción es individual, no colectiva, ni institucional.

2. La objeción aplica sólo a prestadores directos y no al personal administrativo sanitario.

3. Quien objete debe asegurar la remisión a un prestador disponible, a través de una lista de proveedores públicos y privados que estén disponibles.

4. La objeción debe expresarse en forma previa y completa: no durante el proceso de atención o prestación del servicio y sobre todo, de manera asertiva sin promover la discriminación, acoso o invalidación de los deseos del paciente o sus familiares.

5. La objeción es un procedimiento que debe hacerse por escrito y estar fundamentada en razones y convicciones íntimas y profundas de carácter moral, ético y religioso (si es el caso).

6. Quienes objetan no pueden ser discriminados y tampoco quienes presten servicios objetados.

Ya se ha señalado que la objeción de conciencia es un derecho fundamental, pero que en ningún sentido modifica la obligación ética que se tiene en la profesión de no interferir con la autonomía de los pacientes, de salvaguardar su bienestar y, más aún, de no hacer daño o negar información importante. ${ }^{9}$ Por tanto, la objeción de conciencia es una excepción más que una práctica generalizada que posee como finalidad el cumplimiento de las obligaciones científicas, bajo el estricto cuidado y consideración para el reconocimiento integral a la dignidad humana de todos los involucrados.

\section{CONCLUSIONES}

La objeción de conciencia en sí misma no representa la solución a los extraordinarios dilemas bioéticos que enfrenta el país en la actualidad. Por ello, es fundamental la creación de estructuras jurídicas que den sustento a sus promulgaciones generales, de tal forma que no sólo exista el reconocimiento de estos derechos, sino que también se facilite el acceso a los mismos, estableciendo guías de acción claras y puntuales para que se ejerza y proteja el derecho en cuestión. ${ }^{10}$

El análisis actual señala que existe un evidente conflicto entre la objeción de conciencia con otros bienes y derechos fundamentales, que resultan irremediablemente dañados cuando persiste la actitud del objetor. Sin embargo, la regulación de este acto con el reconocimiento de sus alcances y limitaciones dan pie a la configuración de una metodología específica que señala el camino legal y ético para que se salvaguarden los derechos no sólo de los pacientes, sino también de sus familiares, y del personal de salud involucrado. Debido a que se ha reconocido nominalmente este derecho en la Ley General de Salud para asegurar su tutela efectiva es crucial delimitar cuál es su preciso contenido, estableciendo pautas de acción que aseguren su cumplimiento en pro del reconocimiento a la dignidad de todos los involucrados y no solamente de algunos de ellos.

Asimismo, los aspectos éticos de las intervenciones sanitarias requieren que los profesionales de la salud interactúen con bioeticistas para analizar las relaciones de riesgo beneficio de sus acciones, además de asegurar la equidad y la justicia de todas ellas. ${ }^{2}$ Por ello, las observaciones en materia de bioética merecen una puntual atención y distribución para fomentar una verdadera comprensión holística que permee en todas las esferas de la protección a la salud, pues, elementos como el manejo de la información, el acercamiento asertivo y el cuidado de la relación médico-paciente que son ejes elementales, deben ser considerados en toda actuación y razonamiento para ser considerados pertinentes.

\section{REFERENCIAS}

1. Penchaszadeh V. Bioética y salud pública, Rev Iberoam Bioética, 2018; 7: 1-15.

2. González-Vélez AC. Objeción de conciencia, bioética y derechos humanos: una perspectiva desde Colombia, Rev Bio y Der, 2018; 42: 105-126.

3. Kraus, A. Diccionario incompleto de Bioética. México: Ed. Taurus; 2012.

4. Cabello-Robertson J, Núñez-Nova A. Objeción de conciencia institucional y regulación en salud; cexiste una excusa legítima frente al aborto en Chile?, Rev Bio y Der, 2018; 43: 161-177.

5. Nazzal O. Objeción de Conciencia, Rev Chil Obstet Ginecol, 2017; 82 (4): 348-349.

6. Pérez D'Gregorio R. Objeción de conciencia, Rev Obstet Ginecol Venez, 2012; 72 (2): 73-76.

7. Bárcena ZA. La objeción de conciencia de los testigos de Jehová en relación con los símbolos patrios en México. Un caso de colisión de principios constitucionales, Isonomía, 2007; 26: 167-217.

8. Alba-Bermúdez JM. La objeción de conciencia en el ámbito sanitario: especial referencia a la legislación ecuatoriana 
[Tesis doctoral]. Escuela Internacional de Doctorado de la Universidad Nacional de Educación a Distancia; 2018.

9. Soto Obregón ME, Ruiz Canizales R. Tratamiento "habitual"; de la objeción de conciencia (oc) y la desobediencia civil (dc) en la teoría del derecho, Temas Socio-Jurídicos, 2013; 31 (63): 16-38.

10. Alegre M. Opresión a conciencia: La objeción de conciencia en la esfera de la salud sexual y reproductiva. SELA (Seminario en Latinoamérica de Teoría Constitucional y Política). 2009; papers: Yale
Law School Legal Scholarship Repository. Available in: https://digitalcommons.law.yale.edu/yls_sela/66/

\author{
Correspondencia: \\ Martha Patricia Hernández Valdez \\ E-mail: martha.hernandez@anahuac.mx
}

